\title{
UN ACERCAMIENTO AL CAPITAL SOCIAL Y AL TURISMO DESDE EL ENFOQUE MIXTO Y MAPEO DE ACTORES
}

\author{
Omar Ismael RAMÍREZ HERNÁNDEZ; Graciela CRUZ JIMÉNEZ; \\ Elva Esther VARGAS MARTÍNEZ \\ Universidad Autónoma del Estado de México \\ omar.ramirez.hdez@hotmail.com,gracicj@hotmail.com, elvacolegio@hotmail.com
}

\section{AN APPROACH TO SOCIAL CAPITAL AND TOURISM USING THE MIXED METHOD AND STAKEHOLDERS MAPPING}

Resumen: La complejidad que reviste estudiar el binomio de capital social y turismo requiere un enfoque integral y metodologías que incluyan a los diferentes actores que forman parte de dicha actividad. En este artículo se propone un acercamiento metodológico basado en el enfoque mixto y en el mapeo de actores para conocer el capital social de los actores sociales, públicos y privados; ya que este tipo de capital puede ser afectado por la actividad turística. A diferencia de otras investigaciones que lo excluyen, la contribución de esta propuesta es integrar al turista como parte del análisis; asimismo, identificar a quienes participan en esta actividad y posicionarlos respecto a ésta. Una de las principales conclusiones es que al tomar en cuenta el punto de vista de todos los actores relacionados con el turismo, se reduce el sesgo del análisis sobre esta actividad, pues las investigaciones sólo se limitan a conocer la perspectiva de un tipo de actor; algunas de ellas el público, otras el privado y unas más el social, pero no los integran a todos en un mismo estudio.

Abstract: The complexity of studying the binomial social capital and tourism requires a comprehensive approach and methodologies that include the different stakeholders which take part in that activity. This article proposes a methodological approach based on the mixed method and stakeholder's mapping to analyze social capital because it could be affected by the tourism. Unlike other research, the contribution of this proposal is to integrate the tourist as part of the analysis and identify those who participate and their relation with this activity. One of the main conclusions is that taking into account the point of view of all stakeholders related to tourism, reduces the bias of the analysis on this area, because other research are limited to knowing the perspective of a type of actor; some of them the public, others the private or the social, but do not integrate all perceptions in the same study.

Palabras clave: Capital Social. Enfoque Mixto. Mapeo. Turismo Social Capital. Mixed Method. Mapping. Tourism 


\section{Introducción}

Aunque la discusión teórica y la aparición del capital social en la literatura especializada son relativamente recientes, este tipo de capital utiliza una noción que no es novedosa para los investigadores, que hace énfasis en las relaciones entre los diversos actores en una sociedad (Sobel, 2002). De esta forma, dicho capital se vincula en términos generales con un cúmulo de relaciones y de recursos de los actores de una comunidad para alcanzar objetivos comunes.

Por otro lado, el turismo, se ha convertido en una de las actividades económicas, sociales y culturales de mayor impacto, por lo que se reconoce su importancia a nivel mundial (Page y Connell, 2006) y a la vez se ha constituido en un campo de estudio emergente. En consecuencia, la comunidad académica ha realizado múltiples aportaciones en torno a sus aristas política, económica, medioambiental, social y cultural. Algunas de ellas abordan los impactos del turismo en los destinos y lo reconocen como un agente de cambio.

No obstante, las investigaciones que retoman de manera conjunta al capital social y al turismo son escasas; aun así, han sido realizadas desde diversos enfoques, lo que denota que la temática es novedosa y tiene un significativo potencial para la producción académica, lo que hace necesario definir una ruta metodológica que aborde este binomio desde una perspectiva más completa.

De forma general, el capital social es considerado como el conjunto de relaciones entabladas por actores sociales, públicos y privados de una sociedad para cumplir y resolver problemas colectivos o particulares. Varios autores (Putnam, 1993; Coleman, 1990; Durston, 2001; Inkpen y Tsang, 2005; Enrique y Rangel, 2008; Bourdieu, 1985) lo han posicionado como un término relevante dentro de la investigación científica. En tal sentido, Woolcock y Narayan (2000), plantean cuatro maneras abordarlo:

- La comunitaria: esta forma identifica los efectos positivos del capital social en las relaciones entre organizaciones locales, como clubes, asociaciones y grupos cívicos de una o más comunidades.

- De redes: aquí destacan la importancia, tanto de las asociaciones verticales como de las horizontales, en el ámbito personal y entre organizaciones de una o más comunidades.

- Institucional: esta forma sostiene que la vitalidad de las redes comunitarias y de la sociedad civil es resultado del contexto político, legal e institucional, donde la acción colectiva de los grupos dependerá de la calidad de las instituciones formales.

- Sinérgica: esta es un intento de integrar las perspectivas institucionales y la de redes, analizando las alianzas profesionales y su relación entre burocracias estatales y diversos actores de la sociedad civil.

En lo que respecta al turismo, es necesario poner de relieve que cualquier intento por abordarlo y por descubrir sus alcances, debe reconocer los elementos que participan en él y que son afectados por éste (Goeldner y Ritchie, 2009: 5). En tal sentido, Wall y Mathieson (2006), argumentan que esta actividad genera importantes impactos socioculturales, ambientales y económicos que afectan a las sociedades, derivado del desplazamiento de millones de personas de su lugar de residencia hacia destinos turísticos.

Si bien el capital social y el turismo tienen ciertas complejidades cuando son abordados de manera particular, al hacerlo conjuntamente dicha condición aumenta, lo cual queda en evidencia en la literatura científica donde existe un debate sobre de los actores que toman parte de esta actividad y las variadas propuestas para estudiar y medir el capital, de acuerdo a los objetivos de cada estudio y a su enfoque. 
En algunas investigaciones prevalece el corte cualitativo (Alvarez, 2005; Richards y Wilson, 2005; Merinero, 2008a; Andrade, 2008; Barbini, 2008; Cacciuto y Barbini, 2012; Park et al, 2012; Mura y Tavakoli, 2014); en otras, el enfoque cuantitativo (Zhao et al, 2011). Otros autores han optado por uno mixto (Hwang y Stewart, 2016); Las entrevistas y la encuesta predominan como herramientas, en tanto que los actores estudiados son los sociales, públicos y privados, pero en la mayoría de casos de manera separada.

Ante la existencia de diversas metodologías que utilizan distintos enfoques, variables y actores para abordarlo, se reconoce la complejidad y la multidimensionalidad para medir el capital social y el turismo. A ello se suma el debate respecto a las distintas propuestas para medirlo según la perspectiva y propósitos de cada estudio. Frente a tal diversidad se reitera la importancia de crear una metodología para abordar al turismo y el capital social, que incorpore la percepción de todos los actores relacionados con esta actividad.

Se pretende que esta propuesta que combina técnicas cualitativas y cuantitativas, basada en un enfoque mixto y complementada con el mapeo de actores, contribuya a entender y a generar alternativas que atiendan los problemas en las comunidades receptoras, con el fin de beneficiar a sus habitantes. Con base en lo anterior, el objetivo de este artículo es plantear una metodología que aborde dicho binomio, identificando a los actores sociales, públicos y privados que participan en la actividad turística del espacio geográfico elegido por el investigador, quien deberá tomar en cuenta sus particularidades para el análisis.

Para atender metodológicamente tal propósito es utilizado un enfoque mixto que permite la combinación de métodos, técnicas y aproximaciones del enfoque cualitativo y cuantitativo; con ello se pretende matizar las desventajas de cada uno, pero sobretodo aprovechar sus ventajas.

Este artículo se deriva de la investigación "Impactos del turismo en el capital social de los actores del turismo en Teotihuacán y San Martín de las Pirámides, México” y se integra por los siguientes apartados: El primero plantea los fundamentos del enfoque mixto, que incluye sus ventajas y desventajas; mientras que el segundo aborda los actores en el turismo y el capital social. Los anteriores elementos sirven como base para plantear la propuesta metodológica basada en el mapeo de actores que posibilite analizar su capital social en los destinos turísticos, lo cual puede apoyar la planificación y gestión de los espacios geográficos donde se practica el turismo.

\section{Fundamentos del Enfoque Mixto}

En términos generales este enfoque es conceptualizado como el uso o la combinación de metodologías de investigación provenientes de las tradiciones cuantitativa y cualitativa (Pole, 2009). Según Terrell (2011), los estudios con métodos mixtos han surgido del paradigma donde se complementan los enfoques cualitativo y cuantitativo.

Hernández et al, (2006) señalan que el cuantitativo usa la recolección de datos para probar hipótesis con base en la medición numérica y el análisis estadístico a fin de establecer patrones en un fenómeno determinado. Dicho enfoque es basado en el positivismo y rechaza toda idea que no sea posible comprobar con hechos factibles; es decir, el conocimiento es validado siempre y cuando se pueda medir (Pereira, 2011).

Monje (2011:13) complementa la idea anterior, pues expone que "para el enfoque cualitativo es esencial prescindir de toda case de enunciados pre-científicos, como las creencias, la percepción subjetiva, los prejuicios y valoraciones que desnaturalizan el conocimiento científico". De esta forma, el investigador debe abstenerse de hacer juicios subjetivos y ser neutral en todo el proceso investigativo.

En contraparte, Taylor y Bogdan (1986) consideran el enfoque cualitativo como aquel que produce datos descriptivos: las palabras de las personas, habladas o escritas y la conducta observable. Trata de evitar la cuantificación y el investigador induce las propiedades del problema estudiado a partir de la forma como orientan e interpretan el fenómeno que se examina. 
De la misma forma, Monje (2011: 14) argumenta que este enfoque busca, de manera sistemática, conceptualizar la realidad con base en el comportamiento, actitudes, valores, creencias de las personas estudiadas. Lo anterior, representa una forma empírica de investigación que complementa la dualidad entre la objetividad y subjetividad, conduciendo éstas hacia una relación dialéctica ${ }^{1}$ donde se analiza parte de una realidad que resulta significativa para los sujetos (Guba y Lincoln, 1994).

Si bien su combinación en las investigaciones científicas da paso al enfoque mixto, Johnson y Turner (2003) argumentan que para desarrollar de manera efectiva este método, es necesario considerar las ventajas más relevantes de ambos (Tabla 1).

Tabla 1 Ventajas de los Enfoques Cuantitativos y Cualitativos

\section{Ventajas Enfoque Cuantitativo Ventajas Enfoque Cualitativo}

- Prueba y valida la construcción de teorías acerca de cómo y por qué ocurren los fenómenos.

- La prueba de hipótesis se construye antes de la recogida de datos. La información obtenida se puede generalizar cuando los datos se basan en muestreo aleatorio de un tamaño suficiente.

- La facilidad para obtener los datos permite hacer predicciones cuantitativas.

- Los investigadores pueden construir situaciones que eliminan la influencia de confusiones de muchas variables.

- Permite mayor credibilidad en la medición de la relación causa-efecto.

- La recogida de datos usando métodos cuantitativos, es relativamente rápida.

- Aporta precisión, cantidad y datos numéricos.

- El análisis de los datos consume menos tiempo.

- Los resultados de la investigación son relativamente independientes del investigador (efecto del tamaño, significación estadística).

- Puede tener una alta credibilidad con pocas personas.

- Es útil para estudiar un amplio número de personas.
- Los datos están basados en categorías de significado.

- Es útil para estudiar en profundidad un limitado número de casos.

- Es útil para describir fenómenos complejos.

- Provee información de casos individuales.

- Puede comparar casos cruzados y análisis.

-Provee una comprensión y descripción de los fenómenos que forman parte de la experiencia personal de la gente.

- Puede describir ricamente detalles, fenómenos, así como su situación y profundidad en el contexto local.

Los investigadores identifican factores contextuales y fijos relacionados con el fenómeno de interés.

- El investigador puede estudiar la dinámica de los procesos.

- El investigador puede usar primariamente el método cualitativo como teoría central y generar inductivamente una teoría tentativa y explicativa acerca del fenómeno.

- Puede determinar cómo los participantes "interpretan constructos".

- Los datos usualmente son recogidos de modo natural.

- La aproximación cualitativa responde a situaciones locales, condiciones y necesidades.

Fuente: Johnson y Turner (2003).

La adecuada combinación de ambos enfoques propiciará que el trabajo genere mejores resultados en comparación si se usara uno solo. Adicionalmente, Tashakkori y Teddlie

1 La dialéctica es un método de pensamiento y de interpretación, que permite una explicación del mundo tanto como de la naturaleza y de la sociedad (Gurvitch, 1971). 
(2008) argumentan que el empleo de ambos ayuda a corregir los sesgos propios de cada enfoque. Adicionalmente Johnson y Onwuegbuzie (2004) identifican algunas ventajas y desventajas en su uso (Tabla 2).

\section{Tabla 2 Ventajas y Desventajas del Enfoque Mixto}

\begin{tabular}{|c|c|}
\hline Ventajas & Desventajas \\
\hline $\begin{array}{l}\text { - Las palabras, dibujos y la narrativa pueden uti- } \\
\text { lizarse para agregar significado a los números. } \\
\text { - Los números pueden usarse para agregar preci- } \\
\text { sión a las palabras, dibujos y a la narrativa. } \\
\text { - Puede proveer las fortalezas de los métodos } \\
\text { cuantitativos y cualitativos. } \\
\text { - Los investigadores pueden generar y probar } \\
\text { teorías fundadas. } \\
\text { - Puede responder a un amplio y más comple- } \\
\text { to rango de preguntas de investigación, porque } \\
\text { los investigadores no están confinados a un solo } \\
\text { método o aproximación. } \\
\text { - Un investigador puede usar las fortalezas de } \\
\text { un método adicional para reducir las debilidades } \\
\text { en otro método utilizando ambos en un estudio. } \\
\text { - Puede proveer fuertes evidencias para una } \\
\text { conclusión mediante la convergencia y corrobo- } \\
\text { ración de la información obtenida en la investi- } \\
\text { gación. } \\
\text { - Puede agregar un nivel de comprensión al es- } \\
\text { tudio, que se puede perder cuando se utiliza un } \\
\text { sólo método. } \\
\text { - Puede usarse para incrementar la generaliza- } \\
\text { ción de resultados. } \\
\text { nocios miento más completo, que necesariamente } \\
\text { informa de la teoría y la práctica. }\end{array}$ & $\begin{array}{l}\text { - Puede ser difícil para un solo investigador } \\
\text { desarrollar ambos métodos, especialmente si } \\
\text { se espera usar dos o más aproximaciones, fre- } \\
\text { cuentemente; esto puede requerir un grupo de } \\
\text { investigadores. } \\
\text { - Los investigadores han de aprender acerca de } \\
\text { múltiples métodos y aproximaciones, y com- } \\
\text { prender cómo combinarlos apropiadamente. } \\
\text { - Los puristas metodológicos concuerdan que } \\
\text { siempre se debe trabajar en un solo métodos y } \\
\text { no en ambos. } \\
\text { - Es más caro. } \\
\text { - Consume más tiempo. } \\
\text { - Es necesario que los investigadores tengan en } \\
\text { cuenta desde el inicio algunos detalles del méto- } \\
\text { do mixto de investigación, como el problema de } \\
\text { combinar paradigmas; cómo analizar los datos } \\
\text { cuantitativos y cualitativos, y cómo interpretar } \\
\text { el conflicto de resultados. }\end{array}$ \\
\hline
\end{tabular}

Fuente: Johnson y Onwuegbuzie (2004).

De esta forma, Johnson y Onwuegbuzie (2004) sostienen que todo enfoque tiene limitaciones, pero la combinación de métodos y técnicas de las dos aproximaciones clásicas puede neutralizar o bien cancelar las desventajas de cada uno. A la vez, como una forma de procurar un mejor acercamiento, se propone una postura transformativa-emancipatoria, por aportar mejores oportunidades en el análisis del impacto del turismo en el capital social de los actores del turismo. En este contexto es importante reconocer que cada uno de ellos tendrá su propia perspectiva con respecto al fenómeno estudiado. 
La naturaleza del tema que se aborda (compleja, relevante, rica en matices, conceptos e ideas, y multidisciplinar), sugiere una combinación de métodos y técnicas cuantitativas y cualitativas. En consecuencia, las variables seleccionadas para conocer el capital social son: redes (estructura social), compromiso cívico, confianza y acción colectiva. De esta forma, el análisis de la primera variable (redes o estructura social) es llevado a cabo a partir del Mapeo de actores, que en términos generales es una representación gráfica de las relaciones entre actores en una sociedad. Por tanto, es necesario conocer quiénes participan en la actividad turística.

\section{Capital Social, turismo y sus actores interesados}

En 1984 el enfoque de los Stakeholders o actores interesados cobró importancia en la investigación científica, gracias a Freeman, quien propuso como parte fundamental de este concepto los siguientes elementos: estrategias, ética empresarial y responsabilidad social corporativa. Por ello, la definición más común y citada sobre el tema corresponde a este autor; él argumenta que cualquier grupo o individuo que puede afectar o ser afectado por el cumplimiento de los objetivos de una organización, puede ser denominado como Stakeholder (Freeman, 2005). A partir de ello, se resalta la importancia de identificarlos por jugar un papel determinante en el desarrollo de cualquier actividad social (Aas et al, 2005).

De esta forma, en el caso del turismo los actores están dentro de la dinámica social y es posible encontrar relaciones entre ellos (Kimbu y Ngoasong, 2013). Por tanto es relevante identificar quiénes influyen e interactúan en la actividad turística, con el fin de comprender cómo ésta ha beneficiado o perjudicado su capital social; ello hace necesario tener en cuenta al menos dos particularidades del turismo: 1) el desplazamiento generado por una persona o grupos de personas fuera de su residencia habitual hacia un destino 2) los encuentros y las relaciones entabladas entre los visitantes y los actores pertenecientes a un territorio.

Adicionalmente, Prandi (2007: 122-123) formula algunas recomendaciones que ayuden a identificar a los stakeholders en el turismo:

- Tener en cuenta no sólo los intereses tangibles o cuantificables, sino también los valores intangibles que existen en las relaciones de cada sociedad.

- No descartar a los stakeholders que puedan ser considerados en primera instancia como poco importantes, pues tal vez por motivos culturales, religiosos o de género, tengan más dificultades para expresar sus preocupaciones, sugerencias o preferencias.

- Valorar la información que intercambian los stakeholders, poniendo atención a las demandas que formulen entre ellos mismos, a fin de identificar los intereses de cada individuo, permitiendo entender sus comportamientos sociales.

- Tener en cuenta que ningún actor se sitúa en el centro de las relaciones, sino que es uno más entre la sociedad.

Bajo esta visión, Merinero (2008b: 186) define a un actor turístico como "aquella persona física u organización que con su acción interviene, directa o indirectamente, en la generación de impactos". Siguiendo esta postura, Merinero y Zamora (2009) identifican como los principales actores vinculados al turismo a las administraciones públicas, empresas de servicios turísticos, grupos de interés empresarial, grupos de interés sindical, personas con recursos turísticos, personas que generan opinión o alguna influencia, el sector privado, organizaciones de la sociedad civil, comunidades locales e, incluso, los propios turistas.

Ying y Zhou (2007) plantean que los actores pueden desglosarse en dos grandes grupos. El primero, conformado por los turistas pasados, actuales y potenciales. El segundo, los actores sociales, privados y públicos cuyo principal objetivo es ofertar y desarrollar el 
turismo en un determinado territorio. De esta forma, ambos grupos crean sus redes sociales alrededor de actividades de este tipo o participan en vínculos creados con respecto al turismo, estableciendo un cierto nivel y tipo de capital social. Por lo tanto, cuando los dos grupos anteriores entablan relaciones, dicho rubro será el causante de generar cambios en las condiciones socioculturales, económicas, políticas, ambientales de otros actores sociales (incluyendo a los turistas).

Por otro lado, Massey (2009) argumenta que destacan cuatro actores con amplia capacidad para influir y configurar el destino: la comunidad local, el sector público, el sector privado y los turistas; aunque el autor hace hincapié en que estos últimos no forman parte del destino, sí califica de significativo el poder de apropiación simbólica que ejercen en dicho espacio.

En tal contexto, Pulido (2010) expone la necesidad de agrupar a los stakeholders para entender sus relaciones, pero al mismo tiempo para lograr una comprensión más amplia del turismo, partiendo de los actores interesados que lo conforman y cómo éstos se relacionan en un entorno social, económico, ambiental y político desde una perspectiva social; lo anterior en aras de facilitar el entendimiento de los impactos que provoca dicha actividad.

Por lo anterior, Morales y Hernández (2011) evidencian la diversidad de actividades que comprende el turismo, lo cual, hace que identifiquen un número diverso de actores que funcionan independientemente unos de otros, pero que tienen en común la actividad turística. En tal sentido, estos autores identifican a las siguientes partes interesadas en un destino turístico; 1) Empresas de alojamiento (hoteles, hostales, casas rurales, campings, apartamentos turísticos, etc.); 2) restaurantes; 3) Touroperadores, agencias de viajes mayoristas y minoristas o empresas de intermediación; 4) Centrales de reservas; 5) Empresas de Actividades Turísticas; 6) Museos, Teatros y Centros de Interpretación; 7) Oficinas de Turismo; 8) Asociaciones y clubs deportivos, culturales y de ocio (Asociaciones de senderismo, micológicas, cinegéticas, ornitológicas o teatrales, entre otros, dependiendo de las distintas tipologías de turismo existente); 9) Ayuntamientos, Mancomunidades, Grupos de Acción Local, Diputaciones Provinciales, Consejerías, Ministerios y demás organismos públicos relacionados, en función de las tipologías de turismo existentes; 10) Fundaciones, Universidades y otros organismos relacionados y con carácter relevante en el sector; 11) Edificios y lugares de interés turístico y cultural; 12) Espacios naturales protegidos, Patrimonios de la Humanidad y demás figuras de interés; 13) Periodistas y medios de comunicación especializados; 14) Promotores del destino en el extranjero; 15) Empresas del sector del comercio; 16) Empresas del sector del transporte terrestre y marítimo.

Estos actores expuestos por Morales y Hernández son considerados para este acercamiento metodológico; sin embargo, para clasificarlos se retoma la propuesta de Goeldner y Ritchie (2009), quienes los concentran en cuatro grandes grupos: turistas, comunidad local, empresas privadas y gobierno.

Asimismo, dado que el capital social engloba solo a los actores sociales, privados y públicos, se adapta la propuesta de Goeldner y Ritchie, agrupando en actores sociales a la comunidad local y al turista; en actores privados a las empresas privadas; y en actores públicos al gobierno e instituciones públicas. A continuación se detalla quiénes corresponden a cada uno de ellos.

\subsection{Actores Sociales}

\subsubsection{La comunidad local}

Mason (2003), menciona que los términos más apropiados para definir a los grupos sociales de un destino turístico son: comunidad local, comunidad residente o comunidad de destino. Singh et al, (2003) mencionan que comunidad puede concebirse como la gente que vive en un mismo espacio, comparte símbolos y personalidades representativas para 
una colectividad. Por su parte, Jafari (2000) la define como una combinación de unidades y sistemas sociales, dados por aspectos como: zona geográfica, interacción social y normas comunes de conducta, con la finalidad de conseguir: economía exitosa, la socialización, el control social, la participación social y el apoyo mutuo.

En este marco es muy importante resaltar que por una comunidad no sólo se debe entender un espacio geográfico, sino también un grupo de personas que viven en él, de tal manera que ellos se identifiquen con sus propias costumbres, creencias, forma de vestir, lenguaje o dialecto, etc. De acuerdo con Monterrubio (2009), desde una perspectiva antropológica, la comunidad local abarca el espacio geográfico, sus habitantes y sus características; de igual manera las manifestaciones culturales y sociales que se generan entre los habitantes, además de las relaciones entabladas por ellos.

En sintonía con este planteamiento, Fernández y González (2006) entienden como comunidad local, un grupo de personas que conviven en un espacio geográfico determinado, que comparten intereses, creencias, sentimientos, valores, actitudes, disposiciones y expectativas que poseen un grado de organización y pueden llegar a compartir un gobierno y, se diferencian de los demás por una herencia histórica y cultural que comparten. Esta definición atiende en su mayoría al concepto que debería tenerse con respecto a una comunidad, ya que toma en cuenta los elementos geográfico, cultural, social y antropológico que la integran. Por tanto, se retoma este concepto de Fernández y González.

De tal forma, para los propósitos de esta propuesta metodológica, los actores sociales que pertenezcan a la comunidad local, deberán residir en el espacio de estudio, compartir intereses, creencias, sentimientos, valores, actitudes, disposiciones, expectativas y una herencia histórica que pueda diferenciarlos de otras comunidades.

Por otra parte, la comunidad local, por ser parte fundamental del turismo, suele estar expuesta a posibles cambios, tanto sociales, económicos y culturales, debido a su interacción con el turista o visitante (Mason, 2003). En consecuencia, es fundamental definir al otro actor social: el turista.

\subsubsection{El turista}

Al visualizar al turismo desde una perspectiva económica, el turista es sinónimo de la demanda, que ha sido utilizada generalmente para referirse al visitante temporal que permanece al menos veinticuatro horas en el país que visita; las finalidades de su viaje pueden clasificarse en: placer, distracción, vacaciones, salud, instrucción, religión y deportes, negocios, familia, misiones y reuniones (Wall y Mathieson, 2006). Asimismo existen varias características que deben ser destacadas y que son indispensables para definir al turista. Quesada (2010) menciona las siguientes:

- Turista es quien viaja a otro lugar distinto al de su domicilio y después regresa al mismo.

- Se considera la duración del desplazamiento, así como la permanencia en el destino, donde debe ser mayor a 24 horas y menor a un año, por lo que debe existir una pernoctación.

- Los propósitos del viaje (motivaciones y motivos) son muy amplios y diversos.

- No se hace diferenciación por el sexo, la raza, idioma, ocupación, edad, país de origen, etc.

- No se consideran como turistas aquellos que viajen con motivos de conseguir un empleo, fijar su residencia en el destino, inmigrantes, diplomáticos, representantes consulares, miembro de fuerzas armadas, refugiados, 
trabajadores fronterizos y pasajeros de tránsito (a menos que permanezcan uno o más días).

Como parte de esta propuesta metodológica, el turista es reconocido como "aquella persona que realice un desplazamiento fuera de su residencia habitual con diferentes motivos y motivaciones de viaje, sin intención de pertenecer a la población de un destino". Por tanto, los actores sociales que sean considerados como turistas, deberán desplazarse desde su lugar de residencia habitual por diferentes motivos y motivaciones, sin intención de quedarse a vivir en el destino.

\subsection{Actores Privados}

Otro de los actores que se incorporan para para este documento son los privados; en general, los constituyen las empresas u organizaciones creadas por capital financiero particular (Merinero y Zamora, 2009). En este sentido, el turismo desde su perspectiva económica, se vincula con diferentes tipos de empresas, que incluyen entre otras:

- Empresas de alojamiento (hoteles, hostales, casas rurales, campings, apartamentos turísticos, entre otras empresas; las cuales ofrezcan el servicio de arrendamiento de habitaciones).

- Empresas de alimentos y bebidas (restaurantes, cafeterías, fondas, taquerías, entre otros).

- Touroperadores, agencias de viajes mayoristas y minoristas o empresas de intermediación.

- Centrales de reservas.

- Empresas de Actividades Turísticas.

- Museos, Teatros y Centros de Interpretación.

- Asociaciones y clubs deportivos, culturales y de ocio (Asociaciones de senderismo, micológicas, cinegéticas, ornitológicas o teatrales, entre otros, dependiendo de las distintas tipologías de turismo existente).

- Fundaciones, Universidades y otros organismos relacionados y con carácter relevante en el sector privado.

- Edificios y lugares de interés turístico y cultural.

- Espacios naturales protegidos, Patrimonios de la Humanidad y demás figuras de interés.

- Periodistas y medios de comunicación especializados.

- Promotores del destino en el extranjero.

- Empresas del sector del comercio.

- Empresas del sector del transporte aéreo, terrestre y marítimo.

Aunque es relevante el número de actores privados vinculados con el turismo, se toman en cuenta aquellos que en palabras de Barros y Santos (2009), resultan fundamentales para tal actividad. Por lo tanto, se considera como actor privado a todas aquellas organizaciones o empresas cuya principal actividad económica dependa del turismo y que además esté situada dentro del destino.

\subsection{Actores públicos}

Por otro lado, figuran los actores públicos, referidos a las organizaciones e instituciones que dependen enteramente del capital que aporta el gobierno. En este sentido, Quintana 
(2014) menciona que la Nación y el Estado juegan un papel importante en todas las actividades sociales y económicas que se desarrollan en un país, ya que buscan regular y satisfacer intereses específicos para los gobernantes.

En dicho marco, el turismo cuenta con la participación de organizaciones e instituciones que ayudan a su desarrollo, crecimiento y normatividad, entre ellas las siguientes:

- Gobiernos Municipales, Estatales y Federales (Ayuntamientos,
Mancomunidades, Grupos de Acción Local, Diputaciones Provinciales,
Consejerías, Ministerios y demás organismos públicos relacionados, en
función de las tipologías de turismo existentes).
- Fundaciones, Universidades Públicas con carácter relevante en el sector.

De esta forma, para esta propuesta metodológica, los actores públicos son todas aquellas instituciones creadas por el gobierno, cuyo fin sea gestionar, legislar y gobernar todos los recursos presentes en el destino turístico.

Aunque generalmente es alto el número de quienes intervienen en la actividad turística, identificarlos a todos o a la gran mayoría, permitirá entender cómo han cambiado las relaciones entre ellos. Asimismo, es vital recordar que si uno de los actores relacionados con la actividad turística sufre una perturbación, no lo afectará sólo a él, también al resto, casi siempre con resultados múltiples e inesperados (Farrel y Twining-Ward, 2004).

Una vez expuesto el tema de los actores relacionados al turismo, se plantea la propuesta metodológica orientada a conocer cómo el turismo influye en el capital social de los actores relacionados con él, incorporando al análisis a todos los sectores que forman parte de este proceso, no de manera parcial como lo han hecho otras investigaciones. Como parte de esta aproximación metodológica destaca el mapeo de actores, herramienta elegida por su importancia al explicar la estructura social de comunidades. Para el resto de elementos del capital social (compromiso cívico, confianza y acción colectiva), se proponen la entrevista y la encuesta.

\section{Mapeo de actores}

El análisis desde la perspectiva de los actores ha sido ampliamente utilizado en las ciencias sociales con diferentes objetivos. Según Gutiérrez (2001), el mapeo de actores es la representación de la estructura social en un esquema, identificando elementos para su comprensión y solución de problemas.

En este sentido, los investigadores han generado métodos y técnicas específicas para analizar la estructura social y todo lo que ésta implica. Reconocen la importancia de esta herramienta al explicar la estructura social de comunidades.

La literatura científica asume distintas denominaciones para los acercamientos de esta naturaleza: uno de ellos es el análisis de redes (Wasserman y Faust, 1994; Haugdahl, 1999; Boccaletti et al, 2006), centrado en las relaciones entre actores, proporcionando herramientas tanto visuales como matemáticas para su entendimiento. Otra denominación es el análisis de actores (Baker, 1990; Burt, 1997; Burgoyne, 2004) enfocado en determinar los intereses, poder e influencia de actores específicos respecto a una actividad. En tanto, el mapeo de actores (Clarkson, 1995; Fletcher, et 1, 2003; Dong et al, 2014) retoma las características de las anteriores, analizando tanto al actor como las relaciones que éste entabla con otros. Esta aproximación reconoce además las redes, estructura y valor de cada actor relacionado con una actividad específica, en este caso la turística.

Sanz (2003) argumenta que este mapeo es un conjunto de técnicas de análisis para el estudio formal de las relaciones entre actores y el análisis de las estructuras sociales que surgen de la recurrencia de esas relaciones o de la ocurrencia de determinados eventos. En 
concordancia con él, Molina (2001) sostiene que no sólo consiste en un listado de posibles actores y sus relaciones en un territorio, sino en conocer sus acciones, sus valores, los objetivos del por qué están en un lugar determinado y su perspectivas en un futuro inmediato. Ambos autores coinciden en cuanto a que el mapeo va más allá de identificar a los actores, sino de establecer su participación en la dinámica social, lo cual aporta una perspectiva más amplia de la realidad. Gutiérrez (2001) resalta las siguientes ventajas de utilizar este tipo de mapeo en investigaciones de corte social:

- Es posible pensar en la sociedad a través de una estructura.

- Las estructuras sociales se manifiestan en forma de relaciones entre actores sociales, públicos y privados (sean éstos individuos, grupos, organizaciones o instituciones).

- Identifica valores, creencias y objetivos particulares de los diferentes actores.

- Comprensión del comportamiento de los diferentes actores según su rol, posición y poder dentro de una sociedad o grupo social.

- Participación en los procesos de toma de decisiones y generación de respuestas a problemas sociales.

De esta forma, se asume que dicho mapeo ayuda a tener una idea general sobre la estructura social y su complejidad, ya que su propósito es representar gráficamente las relaciones en un momento determinado, con miras a entender, evaluar y/o a transformar la situación encontrada. Aunque existen diversas propuestas para elaborar mapeos de actores, esta investigación realiza una propia, debido al su grado de complejidad y practicidad de los temas a abordar. Para ello, se retoman las variables redes (del capital social) y la relación de los actores con la actividad turística. A continuación se describe el proceso de mapeo:

Especificar el tema: Antes de definir quiénes son las personas, grupos $\mathrm{u}$ organizaciones en los que estamos interesados, se estipula el tema que se abordará.

Identificar los actores: Para realizar esta tarea deben realizarse las siguientes actividades:

Contextualizar el área de estudio.

Listar: Mediante una lluvia de ideas y la revisión de la información contextual, elaborar un listado de todas las personas, grupos y organizaciones que puedan cumplir con alguna de las siguientes características:

- Están siendo o podrían verse afectados por el turismo.

- No están siendo directamente afectados, pero podrían tener un interés en el turismo.

- Poseen información, experiencia o recursos para formular e implementar actividades con relación al turismo.

- Son necesarios para la adopción o implementación de nuevas actividades en el turismo.

- Consideran que tienen derecho a participar en las decisiones relacionadas con el turismo.

Mapear los actores: Éste sirve únicamente para identificar a los actores, su posición y su relación respecto a la actividad turística. La propuesta está dividida en dos: a) Posición de los actores con relación al turismo y b) Relaciones sociales entre actores. De este modo, el primer inciso retoma la posición de los actores respecto a la actividad turística; mientras que el segundo inciso incorpora la variable relacional, pues muestra las relaciones sociales entre los actores, que es una de los elementos para conocer el capital social. 


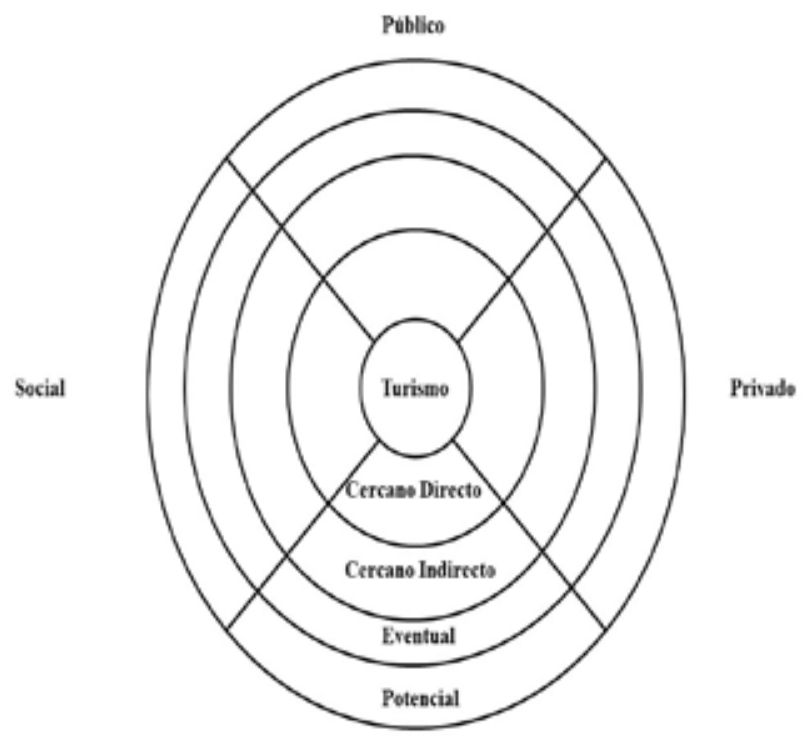

\section{Ilustración 1. Mapeo de actores según su posición y relación con el turismo Fuente: Elaboración Propia.}

Posición de los actores con relación al turismo.- En este apartado se desarrolla la Ilustración 1 que ayuda a posicionar a cada actor según el sector al que pertenezca: público, social o privado y se ubica de acuerdo a su relación con la actividad turística.

A partir de la creación de esta propuesta, se deriva de incluir al turismo, las siguientes cuatro posiciones de los actores en torno a este rubro:

- Cercano directo. Se ubica dentro del destino turístico y mantiene una relación directa con el turismo.

- Cercano indirecto. Se ubica dentro del espacio geográfico del destino, pero no mantiene una relación directa con dicha actividad.

- Eventual. Se puede encontrar en el destino turístico, pero no de manera permanente, pues aunque la relación es directa con la actividad turística, no reside dentro del espacio geográfico del destino.

- Potencial. Corresponde a los individuos y organizaciones que se encuentran fuera del destino turístico; no guardan una relación directa ni indirecta con la actividad, pero tienen la particularidad de que en algún momento pueden insertarse en cualquiera de las tres clasificaciones anteriores.

Relaciones sociales entre actores.- Para atender este punto, se propone un mapa de la estructura social del área de estudio, donde se resaltan las relaciones sociales entre los actores del destino. La Ilustración 2 muestra sólo un ejemplo de la posible visualización.

Los actores fueron identificados con las siguientes figuras geométricas: círculo y triángulo para los sociales (comunidad local y turista), cuadrado para los públicos y rombo para los privados. Para el análisis de dicho mapeo se retoman los indicadores propuestos por Hanneman y Riddle (2001). La tabla 4 muestra el tipo de indicador y su descripción, que ayuda a identificar información importante para explicar la red de vínculos creados por los actores relacionados con la actividad turística. 


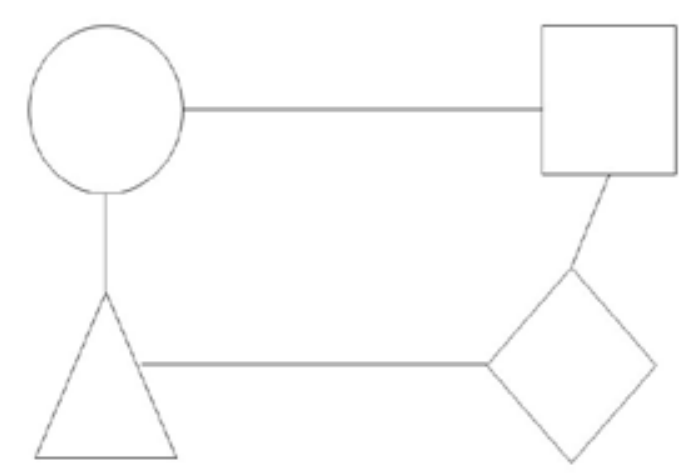

Actores Sociales (Comunidad Local)

- Relacionesentre actores

$\triangle$ Actores Sociales [Turistas)

Actores Públicos

Actores Privados

Ilustración 2. Mapa de relaciones sociales entre actores

Fuente: Elaboración Propia.

Tabla 4 Indicadores para el análisis de redes

\begin{tabular}{|l|l|}
\hline Indicador & Descripción \\
\hline Densidad & $\begin{array}{l}\text { La densidad es una medida expresada en } \\
\text { porcentaje del cociente entre el número de } \\
\text { relaciones existentes con las posibles. Muestra } \\
\text { el valor en porcentaje de la densidad de la red; } \\
\text { es decir, la alta o baja conectividad de la red. }\end{array}$ \\
\hline Centralidad de Grado & $\begin{array}{l}\text { Es el número de actores a los cuales un actor } \\
\text { está directamente unido. }\end{array}$ \\
\hline
\end{tabular}

Fuente: Elaboración propia a partir de Hanneman y Riddle (2001).

A pesar de que para el estudio de una red social existe un sinfín de indicadores, según Muñoz, et al, (2004: 134) "gran parte de los autores que utilizan el análisis de redes sociales; consideran la densidad y la centralidad de grado", por lo que se reconoce para esta propuesta la importancia de dichos indicadores, que sirven para identificar las relaciones sociales que entablan los actores.

Para conocer el resto de variables (confianza, compromiso cívico y acción colectiva) y como éstas han sido influidas por el fenómeno turístico, se requieren la entrevista y la encuesta, ya que permiten obtener información según las características propias de los actores.

\section{Confianza, compromiso cívico y acción colectiva}

Como se planteó en secciones anteriores, esta propuesta parte de un enfoque mixto, ante la complejidad para abordar el binomio turismo-capital social. Las principales variables de 
análisis corresponden a confianza, compromiso cívico y acción colectiva. Para recopilar información sobre ellas, fueron elegidas entre la literatura especializada dos técnicas 1) la entrevista a personas pertenecientes a la comunidad local, a instituciones públicas y a organizaciones privadas 2) la encuesta para recopilar información, únicamente de los turistas. Ambas se describen a continuación.

\subsection{La entrevista}

Ésta permite recopilar información detallada sobre una persona que informa y comparte oralmente con el investigador aquello concerniente a un tema específico (Fontana y Frey, 2005). Heinemann (2003) reconoce las siguientes ventajas al emplearla en la investigación:

- Es posible averiguar hechos no observables como: significados, motivos, puntos de vista, opiniones, insinuaciones, valoraciones, emociones, etc.

- No se somete a limitaciones espacio-temporales, porque es posible preguntar por hechos pasados y también por situaciones planeadas para el futuro.

- Posibilita orientarse hacia un objetivo determinado o centrarlas en un tema específico.

- Permite averiguar tanto informaciones propias (opiniones, motivos, motivaciones del comportamiento, etc.), como observaciones realizadas referentes a un suceso o a otra persona.

La entrevista está fuertemente influenciada por las características personales del entrevistador y del informante; además, se ha convertido en una técnica para abordar diversos temas como el poder, el género, la raza y los intereses de clases, entre otros. Por tanto, como parte de esta propuesta, se sugiere realizarla con actores que se encuentran dentro del espacio geográfico del área de estudio, puesto que le pueden dedicar mayor tiempo al entrevistador.

Es necesario elaborar un guión de entrevista, con el propósito de conocer las variables que constituyen el capital social para el estudio (redes, confianza, compromiso cívico y acción colectiva). A fin de conocer y de retomar aquellas variables pertinentes, el diseño del instrumento debe estar basado en la revisión bibliográfica. En tal sentido, para esta propuesta metodológica el instrumento está compuesto por las siguientes secciones:

- Aspectos sociodemográficos: cada actor detalla sus características principales, como edad, género, actividad relacionadas con el turismo, institución a la que pertenece.

- Redes: se pregunta a cada actor sobre las relaciones que entabla cotidianamente con otros.

- Confianza: se le pregunta cómo el turismo ha influido en la confianza que siente hacia otras personas.

- Compromiso cívico: se pregunta a cada actor sobre el compromiso que siente con otros actores de la comunidad y, cómo el turismo ha influenciado ese compromiso.

- Acción colectiva: se le pregunta sobre las actividades que ha realizado conjuntamente con otros actores y cómo el turismo ha influido en ello.

Con el fin de perfeccionar y validar este instrumento, se requiere realizar un pilotaje con tres actores: uno correspondiente al sector social, otro al público y uno más al privado. Como parte de este ejercicio se verifica la claridad de las preguntas y que las respuestas brinden información relevante para la investigación. 
Después del pilotaje se procede a realizar las entrevistas con los actores relacionados con la actividad turística del área de estudio. Los informantes deben ser elegidos de acuerdo a la referida clasificación (social, público y privado), que incluye a todos los actores vinculados directamente con dicho rubro. Algunas características que deben cumplir los entrevistados son: residir o encontrarse dentro de los dos municipios de estudio y ser mayores de edad según las leyes mexicanas; es decir, tener una edad mínima de 18 años.

\subsection{La encuesta}

La propuesta metodológica que aquí se presenta, incluye la encuesta, definida por Cea (1999: 240) como "la aplicación o puesta en práctica de un procedimiento estandarizado para recabar información (oral o escrita) de una muestra amplia de sujetos. La muestra ha de ser representativa de la población de interés y la información recogida se limita a la delineada por las preguntas que componen el cuestionario precodificado, diseñado al efecto". De esta forma, la autora menciona el uso de procesos estandarizados de interrogación con el propósito de obtener mediciones cuantitativas sobre características objetivas y subjetivas en una población determinada. Adicionalmente, Cea (1999) reconoce sus ventajas:

- Rentabilidad.- permite obtener información diversa, de un amplio sector de la población.

- Fiabilidad.- al ser un proceso estructurado, permite la replicación por parte de otros investigadores.

- Validez.- los resultados obtenidos son de fácil generalización a otras muestras y contextos (suponiendo siempre un alto grado de representatividad de la muestra encuestada).

- Utilidad.- los datos obtenidos gracias a este procedimiento permiten un tratamiento riguroso de la información y el cálculo de significación estadística.

La elección de la encuesta como herramienta para esta propuesta, se basa en estas ventajas, pero también obedece al supuesto de que los turistas en el espacio geográfico del área de estudio elegida, no cuentan con tiempo suficiente que permita el uso de otra técnica.

Se sugiere la elaboración de un cuestionario que sirva de instrumento para conocer cómo el capital social es afectado por el turismo. Las preguntas realizadas deben estar basadas en la revisión bibliográfica, tomando en cuenta las variables de redes: confianza, compromiso cívico y acción colectiva, como elementos que conforman el capital social.

En este sentido, se propone dividir el instrumento en cinco secciones: 1) identificar aspectos sociodemográficos de los turistas, incluyendo su género, edad, nacionalidad, grado máximo de estudios y ocupación 2) la segunda sección está centrada en la variable de redes y se consulta sobre el contacto de los turistas con la comunidad local, con actores públicos, privados y otros turistas.

La tercera, cuarta y quinta sección, incluyen las variables de confianza, compromiso cívico y acción colectiva respectivamente, para las cuales se propone redactar enunciados a fin de conocer cómo el turismo ha influido en estas variables del capital social. Para conocer estas respuestas es recomendable utilizar una escala tipo Likert de cinco puntos, en donde "Totalmente de acuerdo" y "Totalmente en desacuerdo" sean los extremos.

Se sugiere que el instrumento esté redactado en el idioma de origen de los investigadores; sin embargo, dependerá del área de estudio y del contexto de cada investigación, pues en los destinos turísticos existe afluencia de turistas de diferentes nacionalidades y por ende con distinto idioma; por ello es importante adaptar el instrumento a uno entendible para los turistas. 
Asimismo, para la validación del instrumento se propone una prueba piloto que permita identificar si es adecuada su estructura y redacción; además, también el uso de un Alfa de Cronbach (Coeficiente de fiabilidad que proporciona validez y confiabilidad estadística al cuestionario), para posteriormente aplicar la encuesta final.

Manejo y análisis de la información. Se reitera que esta propuesta metodológica utiliza la entrevista y la encuesta como métodos para obtener la información y así posicionar a los actores, además de conocer algunos elementos que componen el capital social (redes, confianza, compromiso cívico y acción colectiva); estas variables fueron elegidas por dos razones: sus ventajas para obtener información sobre los actores y, por las características sociodemográficas de los actores vinculados al turismo.

Con el propósito de dar tratamiento a la información resultante, se propone el uso de algunos programas; la variable de redes puede utilizar UCINET o Pajek, que permiten mapear, editar y analizar redes de relaciones sociales. Mientras que para las otras variables en que se utiliza la escala de Likert, es posible usar el programa Statistical Package for the Social Sciences (SPSS), que facilita analizar la información por medio de frecuencias y cruce de variables.

\section{Conclusiones}

El turismo ha cobrado relevancia debido a las diferentes aristas con que tiene vínculo, lo cual se evidencia en la producción científica que tiene como tema central este campo de estudio. Parte de estas contribuciones corresponden al capital social que apoya el entendimiento de fenómenos sociales. Ante esta complejidad se reitera la importancia de generar una metodología que aborde este binomio, pues el turismo es un agente de cambio que puede cambiar las estructuras sociales de las comunidades receptoras (Mura y Tavakoli, 2014).

Por otro lado, el enfoque mixto se ha constituido como una alternativa para abordar temáticas de investigación en cualquier campo de estudio, porque complementa los enfoques cualitativo y cuantitativo; es por ello, que representa una forma más integral de abordar el binomio capital social y turismo.

Asimismo, el enfoque de los steakeholders parte de la premisa de identificar a los actores interesados en una actividad determinada; por ello, se postula como una forma de identificar a quienes están relacionados con el turismo; junto con el mapeo de actores posibilita posicionar a los actores según la relación que tengan con la actividad turística.

Portes y Landolt (2000) reconocen que tradicionalmente los estudios han abordado por separado el papel del Estado, de las empresas y de las comunidades, sin vincular el papel de cada uno, lo cual limita la información para el entendimiento del capital social. Ante ello destacan la necesidad de tomar en cuenta a todos los actores relacionados en una actividad, a fin de tener una visión general y no sesgada de este tipo de capital.

Adicionalmente, al incluir al turista es posible obtener las visiones de todos los actores relacionados por el turismo, ya que Massey (2009) destaca cuatro actores con amplia capacidad para concebir e influir la actividad turística: la comunidad local, el sector público, el sector privado y los turistas; todos ellos son incluidos en la metodología propuesta.

Por último, se destaca que esta propuesta metodológica es una de las primeras en reconocer de manera conjunta a los actores del turismo y su capital social. Aun así, sus aportaciones son sujetas a mejoras, lo cual deja abierta la puerta a nuevas formas de abordar el binomio turismo y capital social.

\section{Bibliografía}

Aas, C., Ladkin, A., y Fletcher, J. (2005). Stakeholder collaboration and heritage management. Annals of Tourism Research, 32(1), 28-48. https://doi.org/10.1016/j.annals.2004.04.005 
Alvarez, A. (2005). La contribución del turismo al desarrollo integral de las comunidades receptoras. Aspectos Teótico- Metodológicos. Política y sociedad, 42(1), 57-84.

Andrade, M. (2008). Sociedad pesquera y desarrollo turístico pensado desde una Cofradía de Pescadores. Análisis cualitativo de la significación del Proyecto Mardelira. II Jornadas Turismo y sociedad, (págs. 57-84). España.

Baker, W. (1990). Market networks and coporate behavior. American journal of Sociology, 96, 589625.

Barbini, B. (2008). Capacidades locales de desarrollo a travéz del turismo: reflexiones para su abordaje. En Dachary, C. y Burne, A. eds.: Turismo y desarrollo: Crecimiento y Pobreza: 207227. México: Ediciones de la Noche.

Barros, C., y Santos, C. (2009). Earnings of hotel managers: Comparing the human and social capital approach. Tourism Economics, 15(1), 87-103. https://doi.org/10.5367/000000009787536762

Boccaletti, S., Latora, V., Moreno, Y., Chavez, M., y Hwang, D. (2006). Complex networks: Structure and dynamics. Physics Reports, 424|(4-5), 175-308.

Bourdieu, P. (1985). The forms of capital. En Richardson, J. ed.: Handbook of theory and research for the Sociology of Education: 241-258. New York: Greenwood Press.

Burgoyne, J. (2004). Stakeholder analysis. En Cassell, C. y Symon, G. eds.: Qualitative methods in organizational research: a practical guide: 187-207. Londres: Sage.

Burt, R. (1997). A note on social capital an network content. Social Networks, 19, 355-373. https:// doi.org/10.1016/s0378-8733(97)00003-8

Cacciuto, M., y Barbini, B. (2012). Cultura y capital social. EL caso de la comunidad italiana del barrio Puerto de Mar del Plata. Estudios y Perspectivas en Turismo, 21, 681-705.

Cea, M. (1999). Metodología cuantitativa. Estrategias y técnicas de investigación social. Madrid: Visor.

Clarkson, M. (1995). A stakeholder framework for analyzing and evaluating corporate social performance. Academy of Management Review, 20(1), 92-117. https://doi.org/10.2307/258888

Coleman, J. (1990). Foundations of social theory. Cambridge: Harvard University Press.

Dong, S., Burritt, R., y Quian, W. (2014). Salient stakeholders in corporate social responsibility reporting by Chinese mining and minerals companies. Journal of Cleaner Production, 84(1), 59-69. https://doi.org/10.1016/j.jclepro.2014.01.012

Durston, J. (2001). Capital Social: Parte del problema, parte de la solución. En busca de un nuevo paradigma: capital social y reducción de la pobreza en América Latina y el Caribe (págs. 1-43). Santiago de Chile: Comisión Económica para América Latina y el Caribe.

Enrique, J., y Rangel, S. (2008). Capital social: Una Revisión del concepto. Revista CIFE(13), 250263.

Farrel, B., y Twining-Ward, L. (2004). Reconceptualizing tourism. Annals of tourism research, 31(2), 274-295. https://doi.org/10.1016/j.annals.2003.12.002

Fernández, J., y González, A. (2006). Comunidad local y participación política. Articulación necesaria en la construcción socialista. III Conferencia Internacional "La obra de Carlos Marx y los desafios del siglo XXI. La Habana.

Fletcher, A., Guthrie, J., Steane, P., Roos, G., y Pike, S. (2003). Mapping stakeholder perceptions for a third sector organization. Journal of Intellectual Capital, 4(4), 505-527. https://doi. org/10.1108/14691930310504536

Fontana, A., y Frey, J. (2005). The Interview, from neutral stance to political involment. En Denzin, N. y Lincoln, S. eds.: The Sage Handbook of Qualitative Research: 695-727. Londres: Sage.

Freeman, R. (2005). Stakeholder Theory. En Werhane, P. y Freeman, R. eds.: The Blackwell encyclopedia of management, vol. II: 496-500. Oxford: Blackwell Publishing.

Goeldner, C., y Ritchie, B. (2009). Tourism: Principles, Practices and Philosophies. New Jersey: Jhon Wiley \& Sons, Inc.

Guba, E., y Lincoln, Y. (1994). Competing paradigms in Qualitative Research. En Denzin, N. y Lincoln, S eds.: Handbook of qualitative research: 105-117. Thousand Oaks: Sage.

Gurvitch, G. (1971). Dialéctica y Sociología. Madrid: Alianza.

Gutiérrez, P. (2001). Mapas sociales: método y ejemplos prácticos. Diálogos: educación y formación de personas adultas(25), 26-36. 
Hanneman , R., y Riddle, M. (2001). Introduction to social network methods. USA: University of California.

Haugdahl, J. (1999). Network analysis and troubleshooting. USA: Addison-Wesley.

Heinemann, K. (2003). Introducción a la metodología de la investigación empírica en las ciencias del deporte. Barcelona: Paidotribo.

Hernández, R., Fernández, C., y Baptista, P. (2006). Metodologia de la Investigación. México: McGraw Hill.

Hwang, D., y Stewart, W. (2016). Social capital and collective action in rural tourism. journal of Travel Research, 1-13. https://doi.org/10.1177/0047287515625128

Inkpen, A., y Tsang, F. (2005). Social capital, networks, and knowledge transfer. Academy of Management Review, 30(1), 146-165.

Jafari, J. (2000). Enciclopedia del turismo. Madrid: Ed. Síntesis.

Johnson, B., y Turner, L. (2003). Data collection strategies in mixed methods research. En Tashakkori, A. y Teddlie, C eds.: Handbook of mixed methods in social and behavioral research: 297-319. Londres: Sage Publications.

Johnson, R., y Onwuegbuzie, A. (2004). Mixed Methods Research: A Research Paradigm Whose Time Has Come. Educational Researcher, 33(7), 14-26. https://doi. org/10.3102/0013189x033007014

Kimbu, A., y Ngoasong, M. (2013). Centralised decentralisation of tourism development: a network perspective. Annals of Tourism Research, 40, 235-259. https://doi.org/10.1016/j.annals.2012.09.005

Mason, P. (2003). Tourism impacts, planning and management. Oxford: Buttewort Heinemann.

Massey, D. (2009). Concepts of space and power in theory and in political practice. Documents d' Anàlisi Geogràfica, 55, 1526.

Merinero, R. (2008a). Micro-Clúster Turísticos: El papel del capital social en el desarrollo económico local. Revista de Estudios Empresariales. Segunda época(2), 67-92.

Merinero, R. (2008b). Gobernanza, participación de actores y gestión de redes en turismo rural. En Pulido, J. ed.: EL turismo rural. Estructura económica y configuración territorial en España: 185-207. Madrid: Sintesis.

Merinero, R., y Zamora, E. (2009). La colaboración entre los actores turísticos en las ciudades patrimoniales. Reflexiones para el análisis del desarrollo turístico. PASOS REvista de Turismo y Patrimonio Cultural, 7(2), 219-238.

Molina, J. (2001). El análisis de redes social: una introducción. Barcelona: Bellaterra.

Monje, C. (2011). Metodología de la investigación cuantitativa y cualitativa: Guía didáctica. Neiva: Universidad Surcolombiana.

Monterrubio, J. (2009). Comunidad receptora: elemento esencial en la gestión turística. Gestón Turística, 11, 101-11. https://doi.org/10.4206/gest.tur.2009.n11-06

Morales, G., y Hernández, J. (2011). Los stakeholders del turismo. International conference on tourism \& management studies (págs. 894-903). Algarve: Book of Proceedings.

Muñoz, R., Rendon, M., Aguilar, A., García, M., y Altamirano, C. (2004). Redes de Innovación, un acercamiento a su identificación, análisis y gestión para el desarrollo rural. Michoacán: Universidad Autónoma de Chapingo.

Mura, P., y Tavakoli, R. (2014). Tourism and social capital in Malaysia. Current Issues in Tourism, 17(1), 28-45. https://doi.org/10.1080/13683500.2012.718320

Page, S., y Connell, J. (2006). Tourism: A Modern Synthesis. London: Thomson.

Park, D., Lee, k., Choi, H., y Yoon, Y. (2012). Factors influencing social capital in rural tourism communities in South Korea. Tourism Management, 33, 1511-1520. https://doi.org/10.1016/j. tourman.2012.02.005

Pereira, Z. (2011). Los diseños de método mixto en la investigación en educación: Una experiencia concreta. Revista Electrónica Educare, XV(1), 15-29.

Pole, K. (2009). Diseño de metodologías mixtas. Una revisión de las estrategias para combinar metodologías cuantitativas y cualitativas. Renglones, revista arbitrada en ciencias sociales y humanidades(60), 37-42.

Portes, A., y Landolt, P. (2000). Social Capital: Promise and Pitfalls of its Role in Development. Journal of Latin Studies, 32, 529-547. https://doi.org/10.1017/s0022216x00005836 
Prandi, M. (2007). La gestión de los derechos Humanos en la Empresa. Documentación Social, 146, 111-127.

Pulido, J. (2010). Las partes interesadas en la gestión turística de los parques naturals andaluces. Identificación de interrelaciones e intereses. Revista de Estudios Regionales, 88, 147-175.

Putnam, R. (1993). Making Democracy Work: Civic traditions in moder Italy. Princeton: Princeton University press.

Quesada, R. (2010). Elementos de Turismo: teoría, Clasificación. San José: EUNED.

Quintana, R. (2014). Actores sociales rurales y la nación mexicana frente a los megaproyectos mineros. Revista Problemas del Deesarrollo, 45(179), 159-180.

Richards, G., y Wilson, J. (2005). Social capital, cultural festivals and tourism in Catalunya. Anuario Turismo y Sociedad(4), 170-181.

Sanz, L. (2003). Análisis de Redes sociales o como representar las estructuras sociales subyacentes. Apuntes de ciencia y tecnología(7), 21-29.

Singh, S., Timothy, D., y Dowling, R. (2003). Tourism and Destination Communities. En Singh, S. Timothy, D. y Dowling, R. eds.: Tourism in Destination Communities: 3-18. Londres: CABI Publishing.

Sobel, J. (2002). "Can we trust social capital?". Journal of economic literature, 40, 139-154. https:// doi.org/10.1257/0022051027001

Tashakkori, A., y Teddlie, C. (2008). Introduction to mixed method and mixed model studies in the social and behavioral science. En Plano-Clark, V. y Creswel, J. eds.: The mixed methods reader: 7-26. Londres: Sage Publications.

Taylor, S., y Bogdan, R. (1986). Introducción a los métodos cualitativos de investigación. México: PAIDÓS.

Terrell, S. (2011). Mixed-methods research methodologies. The Qualitative Report, 17(1), 254-280.

Wall, G., y Mathieson, A. (2006). Tourism: Changes Impacts and Opportunities. Essex: Pearson Prentice Hall.

Wasserman, S., y Faust, K. (1994). Social network analysis: Methods and Applications. Cambridge: Cambridge University Press.

Woolcock, M., y Narayan, D. (2000). Capital social: Implicaciones para la teoria, la investigación y las políticas sobre desarrollo. Washington: Documento del Banco Mundial.

Ying, T., y Zhou, Y. (2007). Community, goverments and external capitals in China's rural cultural tourism: A comparative study of two adjacent villages. Tourism Management, 28, 96-107. https://doi.org/10.1016/j.tourman.2005.12.025

Zhao, W., Ritchie, B., y Echtner, C. (2011). Social capital and tourism entreprendeurship. Annals of Tourism Research, 38(4), 1570-1593. https://doi.org/10.1016/j.annals.2011.02.006

\section{$\cos$}

